\title{
Image-guided hypofractionated simultaneous integrated boost and adaptive radiotherapy for spine metastasis
}

\author{
Zhen Hou", Shuangshuang Li", Juan Liu, Li Xie, Shanbao Gao, Jing Yan \\ The Comprehensive Cancer Centre of Drum Tower Hospital, Medical School of Nanjing University \& Clinical Cancer Institute of Nanjing \\ University, Nanjing, China \\ Contributions: (I) Conception and design: Z Hou, J Yan; (II) Administrative support: J Yan; (III) Provision of study materials or patients: J Yan; (IV) \\ Collection and assembly of data: S Li, J Liu, S Gao; (V) Data analysis and interpretation: S Li, Z Hou; (VI) Manuscript writing: All authors; (VII) \\ Final approval of manuscript: All authors. \\ "These authors contributed equally to this work. \\ Correspondence to: Jing Yan, MD, PhD. Comprehensive Cancer Centre of Drum Tower Hospital, Medical School of Nanjing University\&Clinical \\ Cancer Institute of Nanjing University, 321 Zhongshan Road, Nanjing 210008, China. Email: yj20030610@126.com.
}

Background: (I) Dosimetric comparison between Helical tomotherapy (HT) and intensity-modulated radiation therapy (IMRT) with hypofractionated simultaneously integrated boost (HF-SIB) technique for spine metastasis. (II) Mega-voltage CT (MVCT) imaging can be used to monitor changes and calculate the true spinal cord dose over multiple fractions, and compare it with the planned dose by deformable image registration.

Methods: We retrospectively analyzed 57 patients with spine metastasis receiving HT at our institution between March 2016 and March 2019. (I) The dose/fraction to planning gross tumor volume (PGTV) and planning target volume (PTV) were 40 or 50 Gy/10 fractions and 30 or 40 Gy/10 fractions. Plans were compared according to PTV coverage and OARs sparing. (II) The daily MVCT were strictly registered with plan CT according to the rigid structures. Contours of the target and critical organs were then deformed from plan CT to MVCT using MIM deformable registration algorithm. After rigid imaging registration, the planning dose matrix was mapped to the MVCT images. The total doses of 10 fractions to the deformed structures were compared with the planned doses.

Results: Comparing HT and IMRT plan averaged over all patients, differences were observed for both homogeneity index $(\mathrm{HI})(\mathrm{P}<0.001)$ and conformity index $(\mathrm{CI})(\mathrm{P}=0.032)$. The maximum dose of the spinal cord was significantly lower in the HT group than the IMRT group $(\mathrm{P}=0.037)$. The actual dose of the spinal cord obtained based on the MVCT modified adaptive radiation therapy (ART) was slightly higher than the initially planned dose, while no significant difference was observed $(\mathrm{P}=0.083)$.

Conclusions: HF-SIB with HT was able to achieve lower spinal cord doses, which was dosimetrically superior to IMRT, especially in those cases where strict control of the spinal cord dose is required. With deformable contours from the planning CT, daily MVCT along with rigid dose transformation can provide quantitative dose guidance for replanning.

Keywords: Helical tomotherapy (HT); hypofractionated; simultaneously integrated boost (SIB); adaptive radiation therapy (ART); spine metastasis

Submitted Jan 02, 2020. Accepted for publication May 14, 2020.

doi: $10.21037 /$ apm-20-13

View this article at: http://dx.doi.org/10.21037/apm-20-13 


\section{Introduction}

Bone metastases present very commonly in the course of solid tumors that can cause pain, spinal cord compression, and pathologic fracture $(1,2)$. About one-third of all bone metastases are located in the spinal column, mostly the lumbar (52\%), thoracic (36\%), and cervical (12\%) spine (3). Conventional radiotherapy has been considered the main treatment options for spinal metastases (4). The main palliative radiation therapy regimen is 30 Gy in 10 fractions, up to $70 \%$ of the patients had some degree of pain relief $(5,6)$. Nevertheless, the commonly used palliative therapeutic options dose was associated with a limited local control (7).

Recently, new innovative treatment schedules such as stereotactic body radiotherapy (SBRT) has been suggested for the patients with spinal metastases $(8,9)$, and outcome data show excellent local control than conventional palliative therapy (10). Due to the spinal cord is the major dose-limiting tissue when increasing the dose of radiotherapy for patients with spine metastasis. Eligible patients with spine bone metastases should continue to be considered for available SBRT (11).

We registered a clinical trial that use of hypofractionated intensity-modulated radiation therapy (IMRT) to dose escalate the tumor volume to 40 Gy would allow for increased local control and palliation of pain symptoms. When patients undergo simultaneously integrated boost (SIB), it is important that the target volume is accurately localized in order to ensure high dose delivery to the target. These anatomic changes and set-up errors during a course of radiation therapy can then possibly cause an unanticipated overdosing to organs at risk (OARs) (12). The dose of OARs we prescribe on the planning CT is not always the dose that the patient receives.

With the development of image-guided adaptive radiation therapy (IGART), its promises improved tumor targeting with smaller margins, and ART further helps in reduction of doses to normal tissues and improves accuracy of delivery (13). Mega-voltage CT (MVCT) imaging can be used to monitor changes and calculate the true spinal cord dose over multiple fractions and compare it with the planned dose by deformable image registration.

Our article recommend the application of our cancer center in precise radiotherapy for spine metastasis, target delineation after CT/PET-CT/MR fusion, dosimetric advantages and monitors with MVCT ART of Helical tomotherapy (HT). We chiefly described (I) a planning studies to compare HT-SIB with IMRT-SIB, and (II) to investigate the discrepancies between the planned doses and daily doses using MVCTs, to determine optimal replanning strategies.

We present the following article in accordance with the STROBE reporting checklist (available at http://dx.doi. org/10.21037/apm-20-13).

\section{Methods}

\section{Patients and tumor characteristics}

From March 2016 to March 2019, 57 patients with symptomatic spine metastases were treated with hypofractionated SIB by HT at our institution. The primary malignancies were including lung cancer, prostatic cancer, breast cancer, esophageal cancer, and others. All the patients need written informed consent. This retrospective study was permitted by the Nanjing Drum Tower Hospital's ethics committee. All the patients were provided written informed consents. Patient general characteristics are described in Table 1.

\section{Simulation and delineation of targets and OARs}

Patients were treated with a supine position and immobilized by a thermoplastic mask. CT images with slice thickness of $3 \mathrm{~mm}$ were obtained and transferred to MIM (MIM Software, Inc., Cleveland, OH). The planning CT images were co-registered to T1-weighted MRI or PET/ CT by using MIM workflow (Figure 1).

The gross tumor volume (GTV) was defined as macroscopic lesion on T1-MRI or pathological uptake on PET-CT. The clinical target volume (CTV) included the entire vertebral body of the involved spine. A margin of $3 \mathrm{~mm}$ was used to determine the PTV. If any epidural or paraspinal soft tissue component of the tumor were present, should also be included. We added $3 \mathrm{~mm}$ safety margin for the spinal cord when the target in close contact with it. We prescribed radiation dose using simultaneous integrated boost technique. PGTV was the boost volume. Other organs, such as spinal cord, lungs, esophagus, trachea, and kidneys were delineated depending on tumor vertebral level.

\section{Treatment planning and acquisition of daily MVCT}

The CT images with delineated structures were transferred to the Tomotherapy planning station (Hi-Art System; Tomotherapy, Madison, WI, USA) and Pinnacle3. Then, the HT-SIB and IMRT-SIB treatment plans for 57 patients 
Table 1 Clinical and histopathological characteristics of the 57 patients

\begin{tabular}{|c|c|}
\hline Characteristics & No. of patients $(n=57)$ \\
\hline \multicolumn{2}{|l|}{ Age (years) } \\
\hline$<65$ & $22(38.6 \%)$ \\
\hline$\geq 65$ & $35(61.4 \%)$ \\
\hline \multicolumn{2}{|l|}{ Gender } \\
\hline Male & 37 (64.9\%) \\
\hline Female & $20(35.1 \%)$ \\
\hline \multicolumn{2}{|l|}{ PS (ECOG) } \\
\hline $0-1$ & $23(40.4 \%)$ \\
\hline 2 & 34 (59.6\%) \\
\hline \multicolumn{2}{|l|}{ Primary tumor } \\
\hline Lung & 16 \\
\hline Breast & 7 \\
\hline Prostatic & 8 \\
\hline Esophageal & 7 \\
\hline Gastric & 5 \\
\hline Colon & $4(23.8 \%)$ \\
\hline Liver & 3 \\
\hline $\mathrm{HNC}$ & 3 \\
\hline Cervical & 2 \\
\hline Other & 2 \\
\hline \multicolumn{2}{|l|}{ Tumor location } \\
\hline Cervical & 16 \\
\hline Thoracic & 21 \\
\hline Lumbar & 20 \\
\hline
\end{tabular}

designed by the physicist. IMRT-SIB was planned using the step-and-shoot technique with 7 coplanar beams. The dose/fraction to planning gross tumor volume (PGTV) and planning target volume (PTV) were 40 or $50 \mathrm{~Gy} / 10$ fraction and 30 or $40 \mathrm{~Gy} / 10$ fraction. The dose prescriptions were designed to cover at least $95 \%$ of the target volume for HT and IMRT plans, respectively. All plans were assessed and confirmed by senior physicians.

Before daily treatment, patient's position was verified by matching the MVCT images to the initial planning CT to minimize interfraction set-up errors. The fractional MVCT images were exported from the Tomotherapy data server and then imported into MIM to analyze the actually dose of critical organs.

\section{Dose calculation using deformable image registration}

MIM Software as a commercially available deformable registration tool is used for this work. Daily MVCT were first rigidly registered with planning CT based on the bony structures. Contours of the target and critical organs were then deformed from planning CT to MVCT using the MIM Software deformable registration tools. After rigid imaging registration, the planning dose matrix was mapped to the MVCT images. The doses to the deformed structures were compared with the planned doses using the Dose Accumulation Deformable workflow. The deformed doses from 10 fraction of treatment are accumulated.

\section{Dose evaluation}

We compared the dose distribution of tumor target and OARs between HT and IMRT groups. Target coverage was analyzed using the homogeneity index (HI) and conformity index (CI). The maximum dose of the spinal cord between the two planning was investigated. The actually total doses were compared with the planned doses using MVCTs.

\section{Statistical analysis}

All statistical analyses were performed using SPSS 16.0 software. The paired t-test was used to compare the dosimetric differences between the two groups. $\mathrm{P}<0.05$ was considered statistically significant.

\section{Results}

Both HT and IMRT plans meet the spinal cord tolerance dose. Dose distributions and dose-volume histograms (DVHs) for all plans were evaluated with the following indices.

\section{Dosimetric comparison for target and organs at risks}

DVHs were generated from each planning group and used to compare target coverage and OARs. The dose distribution of HT and IMRT plan was shown in Figure 2. Dose coverage was expected to be better for HT. Significant differences were observed between the two plans for $\mathrm{HI}(\mathrm{P}<0.001)$ and $\mathrm{CI}(\mathrm{P}=0.032)$ (Table 2). As the OAR were dependent on the location of the spine metastases. Here, we mainly discuss the radiation dose of the spinal cord. The maximum dose of the spinal cord was lower in the HT group than the IMRT group: $33.02 \pm 2.40$ vs. $35.55 \pm 4.16 \mathrm{~Gy}(\mathrm{P}=0.037)$ (Table 2). 

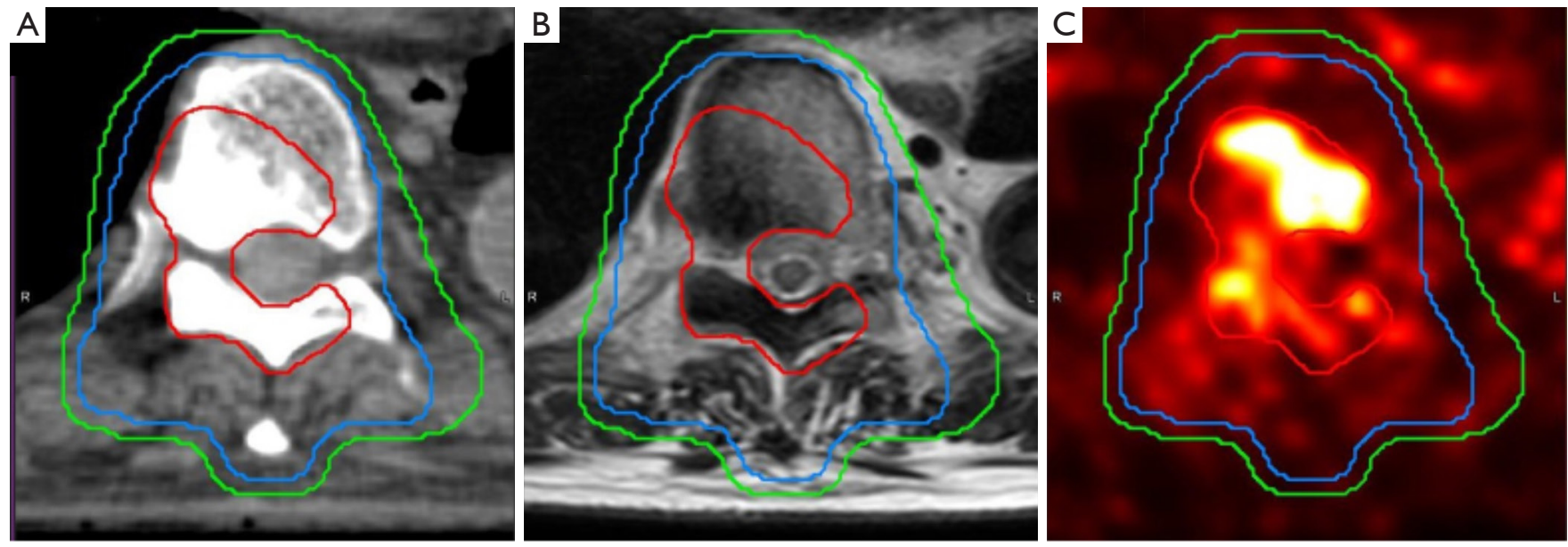

Figure 1 Definition of irradiation targets and OARs. A patient case with prostatic cancer T8 spine metastasis treated with Tomotherapy. The planning CT images (A) were registered to MR (B) and PET/CT (C), respectively. Red line: GTV = PGTV, Blue line: CTV, Green line: PTV.
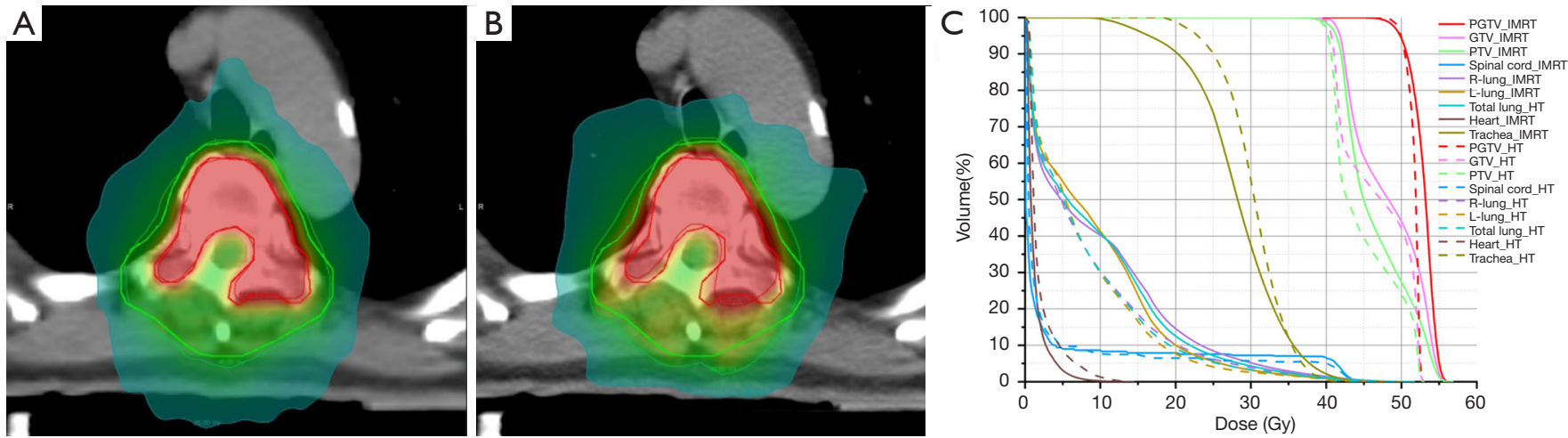

Figure 2 Plan comparison between HT-SIB plan (A) and IMRT-SIB plan (B). Red line: PGTV, Green line: PTV; Red area: 100\% of the prescribed dose, Green area: $80 \%$ of prescribed dose, Blue area: 50\% of prescribed dose. DVHs for the plans above were compared (C). HT, Helical Tomotherapy; SIB, simultaneously integrated boost; IMRT, intensity-modulated radiation therapy; MVCT, Mega-voltage CT; ART, adaptive radiation therapy.

Table 2 The HI, CI of PTV and dosimetric comparison for the spinal cord in two groups (mean \pm SD)

\begin{tabular}{llll}
\hline Parameters & HT-SIB & IMRT-SIB & P value \\
\hline $\mathrm{HI}$ & $0.076 \pm 0.001$ & $0.213 \pm 0.062$ & $<0.001$ \\
$\mathrm{Cl}$ & $0.841 \pm 0.021$ & $0.801 \pm 0.073$ & 0.032 \\
Dose $_{\max }$ of spinal cord (Gy) & $33.02 \pm 2.40$ & $35.55 \pm 4.16$ & 0.037 \\
\hline
\end{tabular}

$\mathrm{HI}$, homogeneity index; $\mathrm{Cl}$, conformal index; HT, Helical Tomotherapy; SIB, simultaneously integrated boost; IMRT, intensity-modulated radiation therapy. 

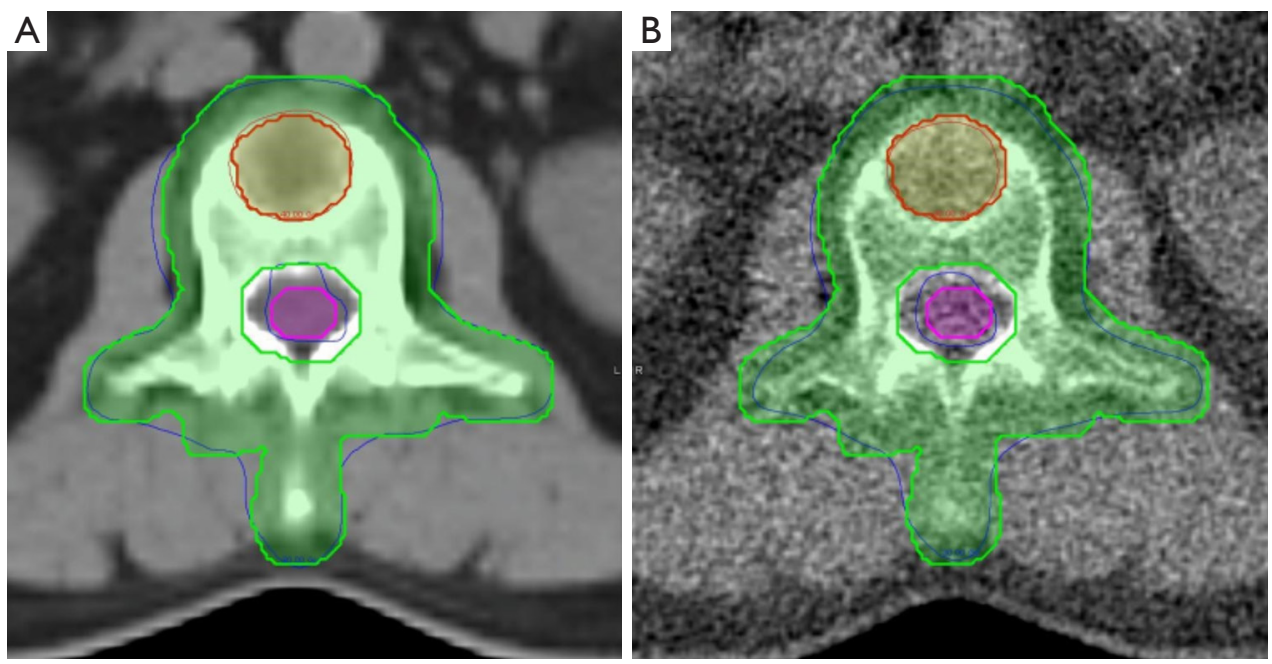

Figure 3 A typical dose distribution of (A) the original HT-SIB plan, and (B) the actual accumulated dose distribution based on the contours projected in the MVCT by modified ART. HT, Helical Tomotherapy; SIB, simultaneously integrated boost; MVCT, Mega-voltage CT; ART, adaptive radiation therapy.

\section{Dose calculation using deformable image registration}

The actual dose of spinal cord obtained based on the MVCT modified ART was slightly higher than the initially planned dose $(33.41 \pm 2.26$ vs. $33.02 \pm 2.40 \mathrm{~Gy})$, but there was no significant differences $(\mathrm{P}=0.083)$. Dosimetric comparisons between the initial planned and the planning MVCT modified ART were shown in Figure 3. The dose distribution of the actual accumulated dose based on the contours projected in the MVCT by modified ART, which is similar for original HT-SIB plan.

\section{Discussion}

For patients with vertebral metastases, local control can be effectively achieved with radiotherapy, especially dose escalations may result in improved tumor control. The use of SBRT has been increasing in the treatment of metastatic spinal tumors. A series of clinical trials reported that about $67.5 \%$ of patients with spine metastases receive SBRT (14). SBRT use for patients who present with spinal cord compression should be considered great caution as absence of a physical separation between the target and adjacent normal critical structures. Eligible patients with spine metastases should continue to be considered for available SBRT trials to clarify the optimal treatment approach. Our study explores hypofractionated SIB technique to spine metastasis that it allows for dose escalation for patients who are not optimal SBRT candidates but may benefit from higher doses than conventional RT. Previous studies verified hypofractionated SIB technique can improve LC compared with conventionally fractionated RT, especially when treating tumors with radioresistant (15).

Our study not only compare dose between HT and IMRT with hypofractionated simultaneously integrated boost (HF-SIB) technique for spine metastasis. To our knowledge, our study is one of the data to use MVCT imaging to monitor changes and calculate the true spinal cord dose over multiple fractions and compare it with the planned dose by deformable image registration. This work is an enlightenment to the coming studies and potentially useful for clinicians in making treatment choices for spine metastasis with higher biological effectiveness.

Both HT and conventional IMRT plans can deliver highquality spine metastasis HF-SIB. Significant differences between HT and IMRT were observed for HI, CI and the max spinal cord dose. HT can achieve more conformed and homogeneous dose distribution, while not increasing the spinal cord dose. Even a circular target could be easily treated with HT using a rotating gantry and could avoid the spinal cord. Our study has allowed clinicians to expand HF-SIB by HT to treat spine metastases close to the spinal cord. In future, we can explore appropriate target area and dose to increase the tumor dose while ensuring the safety of the spinal cord.

Due to weight loss, tumor and normal tissue growth 
or shrinkage, and intra-treatment position variations, the originally planned dose is rarely the same as the actually delivered dose to the patient. Often this will result in the need to create a modified treatment plan, a process known as ART (16). In the ART process, dose accumulation is needed to evaluate the dose already received by the patient at that time during a course of treatment. Image guidance plays an increasingly important role, not only in-patient setup but also in monitoring the delivered dose and adapting the treatment to patient changes (17). Helical Tomotherapy as a novel form of IMRT and IGRT uses daily MVCT imaging to guide the treatment daily. We evaluated the feasibility of a modified ART based on daily MVCT to plan CT using MIM deformable registration algorithm. Our results indicated with deformable contours from the planning CT, daily MVCT along with rigid dose transformation can provide quantitative dose guidance for replanning.

Our findings suggest that most spine metastasis undergoing Tomotherapy do not require ART routinely, because there did not have significant increase in dose to the maximum dose of spinal cord. Nonetheless, treatment replanning was necessary for some patients because of excessive set-up uncertainty and/or weight loss.

\section{Conclusions}

SIB with HT was able to produce highly biological effective dose distributions in the target volumes and minimizing the dose to cord. With deformable contours from the planning CT, daily MVCT along with rigid dose transformation can provide quantitative dose guidance for replanning. The suggested method is sufficiently fast and reliable to be used for daily delivered dose evaluations in clinical application for adaptive tomotherapy of spine metastasis. Through this simple real-time dose monitoring method, we can continue to explore the possibility of increasing the radiation dose in the target area while ensuring the safe dose of the spinal cord.

\section{Acknowledgments}

Funding: None.

\section{Footnote}

Reporting Checklist: The authors have completed the STROBE reporting checklist. Available at http://dx.doi. org/10.21037/apm-20-13
Data Sharing Statement: Available at http://dx.doi. org/10.21037/apm-20-13

Conflicts of Interest: All authors have completed the ICMJE uniform disclosure form (available at http://dx.doi. org/10.21037/apm-20-13). The authors have no conflicts of interest to declare.

Etbical Statement: The authors are accountable for all aspects of the work in ensuring that questions related to the accuracy or integrity of any part of the work are appropriately investigated and resolved. The study was conducted in accordance with the Declaration of Helsinki (as revised in 2013). The study was approved by the Nanjing Drum Tower Hospital's ethics committee (No. ChiCTR1800015955). All the patients were considered to be inoperable and provided written informed consents.

Open Access Statement: This is an Open Access article distributed in accordance with the Creative Commons Attribution-NonCommercial-NoDerivs 4.0 International License (CC BY-NC-ND 4.0), which permits the noncommercial replication and distribution of the article with the strict proviso that no changes or edits are made and the original work is properly cited (including links to both the formal publication through the relevant DOI and the license). See: https://creativecommons.org/licenses/by-nc-nd/4.0/.

\section{References}

1. Coleman RE. Clinical features of metastatic bone disease and risk of skeletal morbidity. Clin Cancer Res 2006;12:6243s-9s.

2. Saad F, Lipton A, Cook R, et al. Pathologic fractures correlate with reduced survival in patients with malignant bone disease. Cancer 2007;110:1860-7.

3. Pilge H, Holzapfel BM, Prodinger PM, et al. Diagnostics and therapy of spinal metastases. Orthopade 2011;40:185-93; quiz 194-5.

4. Chow E, Harris K, Fan G, et al. Palliative radiotherapy trials for bone metastases: a systematic review. J Clin Oncol 2007;25:1423-36.

5. Rades D, Weber A, Bartscht T, et al. A new prognostic factor for the survival of patients with renal cell carcinoma developing metastatic spinal cord compression. Strahlenther Onkol 2014;190:667-70.

6. Chow E, Harris K, Fan G, et al. Palliative radiotherapy trials for bone metastases: a systematic review. J Clin 
Oncol 2007;25:1423-36.

7. Sahgal A, Whyne CM, Ma L, et al. Vertebral compression fracture after stereotactic body radiotherapy for spinal metastases. Lancet Oncol 2013;14:e310-20.

8. Sprave T, Verma V, Förster R, et al. Randomized phase II trial evaluating pain response in patients with spinal metastases following stereotactic body radiotherapy versus three-dimensional conformal radiotherapy. Radiother Oncol 2018;128:274-82.

9. Giaj-Levra N, Niyazi M, Figlia V, et al. Feasibility and preliminary clinical results of linac-based Stereotactic Body Radiotherapy for spinal metastases using a dedicated contouring and planning system. Radiat Oncol 2019;14:184.

10. Chow E, Zeng L, Salvo N, et al. Update on the systematic review of palliative radiotherapy trials for bone metastases. Clin Oncol (R Coll Radiol) 2012;24:112-24.

11. Sahgal A, Larson DA, Chang EL. Stereotactic body radiosurgery for spinal metastases: a critical review. Int J Radiat Oncol Biol Phys 2008;71:652-65.

Cite this article as: Hou Z, Li S, Liu J, Xie L, Gao S, Yan J. Image-guided hypofractionated simultaneous integrated boost and adaptive radio-therapy for spine metastasis. Ann Palliat Med 2020;9(4):1797-1803. doi: 10.21037/apm-20-13
12. Chung Y, Yoon HI, Kim JH, et al. Is helical tomotherapy accurate and safe enough for spine stereotactic body radiotherapy? J Cancer Res Clin Oncol 2013;139:243-8.

13. Pos F, Remeijer P. Adaptive management of bladder cancer radiotherapy. Semin Radiat Oncol 2010;20:116-20.

14. Pan H, Simpson DR, Mell LK, et al. A survey of stereotactic body radiother $\neg$ apy use in the United States. Cancer 2011;11:4566-72.

15. Farooqi A, Bishop AJ, Narang S, et al. Outcomes After Hypofractionated Dose-Escalation using a Simultaneous Integrated Boost Technique for Treatment of Spine Metastases Not Amenable to Stereotactic Radiosurgery. Pract Radiat Oncol 2019;9:e142-8.

16. Schwartz DL. Current progress in adaptive radiation therapy for head and neck cancer. Curr Oncol Rep 2012;14:139-47.

17. de la Zerda A, Armbruster B, Xing L. Formulating adaptive radiation therapy (ART) treatment planning into a closed-loop control framework. Phys Med Biol 2007;52:4137-53. 